\title{
Immunology of Multiple Sclerosis
}

\author{
HENRY F. MCFARLAND
}

\author{
Neuroimmunology Branch \\ National Institute of Neurological and Communicative \\ Disorders and Stroke \\ National Institutes of Health \\ Bethesda, Maryland 20892
}

Although the cause of multiple sclerosis (MS) is not known, an immunologic process is generally considered to be an important element in the pathogenesis of the disease.' Evidence of an immunologic mechanism is however circumstantial. The perivenular inflammatory response comprised of lymphocytes and monocytes is certainly consistent with an immunologically mediated disease and resembles the pathologic changes seen in postvaccinal encephalomyelitis, a disease of certain immunologic cause. The similarities between MS and a model of immunopathologic disease of the central nervous system (CNS), experimental allergic encephalomyelitis (EAE), also supports a similar mechanism in MS. The objections to the appropriateness of EAE as a model for MS have been partially overcome by the demonstration of an experimentally produced relapsing remitting disease with close pathologic similarities to MS. ${ }^{2}$ The relationship between MS and EAE remains uncertain, but it is clear that the induction of MS is considerably more complex.

Further support for an immunologic mechanism in MS comes from the demonstration of abnormalities of immunoglobulin ( Ig) and lymphocyte populations and function in the disease. Alterations in cerebrospinal fluid (CSF) Ig represent the most consistent immunologic changes described in MS, which reflects their diagnostic importance. The increased levels of Ig, largely synthesized locally in the $\mathrm{CSF}^{3}$ and the oligoclonal nature of this $\mathrm{Ig}^{4}$ have led to the contention that MS may be directly related to antibody reactivity with components of myelin. Despite extensive investigations, a clear association between CSF Ig reactivity and the disease has not been established. Increased antibody levels to numerous viruses have been found, and in each case they represent a small fraction of the Ig present. Small amounts of antibody to myelin components have been described, but again it is unlikely that these antibodies are directly or singularly responsible for the demyelination. ${ }^{5}$

An alternative explanation for the increased Ig with multiple specificities is that it merely reflects the specificities of $B$ cells or plasma cells that have migrated into the inflammatory sites within the CNS. The antibody specificities found in the CSF in MS are generally those found in high titers in the serum. This finding suggests that $B$ cells with these specificities have a relatively high precursor frequency and, if circulating, would have a greater chance of migrating into an inflammatory site by chance. Although their accumulation may be random, the conditions permitting their differentiation into Ig-producing cells must be present within the CNS. Because the oligoclonal Ig pattern found in the CSF is often relatively constant over time, it seems likely that some B-cell populations or clones are maintained and undergo continued differentiation within the CNS. Generally, differentiation and $\mathrm{Ig}$ production require two signals, one of which is the perturbation of the $\mathrm{Ig}$ antigen receptor. If the antigens 
to which these antibodies react are not in the CNS, the continued differentiation of $B$ cells must be due to factors in the CNS milieu that are capable of overriding the need for antigen binding to the $\mathrm{Ig}$ receptor. These factors could include lymphokines produced by an ongoing immune response or the presence of neurotransmitters with immunostimulatory activity.

With the exception of CSF Ig abnormalities, evidence of an immunologic process in the pathogenesis of MS points to a cellular immune mechanism. However, there remain fundamental unanswered questions concerning the nature of this process including: What is the mechanism of demyelination? What is the antigen responsible for stimulating this response and where is the antigen presented to T cells? Finally, what accounts for the fluctuating nature of the disease? Although there are no unequivocal answers to these questions, recent studies have begun to provide some insight.

\section{DEMYELINATION}

Several immunologic mechanisms have been considered or suggested as the effector mechanism producing demyelination. These mechanisms include antibody or other serum factors, lymphocytes, particularly cytotoxic $T$ cells, and macrophages. The eloquent ultrastructural studies of Prineas et al. support the supposition that demyelination results from an interaction between myelin and macrophages. This finding is consistent with the observations that macrophages are the predominant cell population in regions of active demyelination. Importantly, macrophages that appear to be stripping myelin were shown to have coated pits. Similar associations between myelin and macrophages with coated pits were described in a relapsing form of EAE in the mouse. ${ }^{2}$ These coated pits most likely represent the migration of receptors within the macrophage membrane. Included among the receptors expressed on macrophages are those of both the Fc portion of Ig and complement. Both groups of receptors are up-regulated with activation of macrophages in sites of inflammation, particularly those associated with delayed hypersensitivity (DTH) reactions.

Either type of receptor could contribute to myelin-macrophage interactions. Antibody to various components of myelin may occur in MS, and antibody bound to myelin could subsequently be bound to $F c$ receptors on activated macrophages. Antibody to galactocerebroside was shown to contribute to the induction of EAE in the guinea pig, ' but it does not seem to be involved in disease production in the mouse or rat. Alternatively, complement may serve as the ligand between myelin and macrophages. Complement could certainly be fixed to antibody attached to myelin, and myelin can bind or fix complement in the absence of antibody. Macrophages express two types of complement receptors: CR 3 and CR $1 .{ }^{8}$ In addition to being up-regulated on activated macrophages, the CR 1 complement receptor becomes mobile in the macrophage membrane and becomes associated with clathin-coated endocytic pits. Consequently, complement could form a bridge between macrophages and myelin by first binding to antibody-coated myelin or directly to myelin.

\section{T-CELL ACTIVATION}

If activated macrophages serve as the final effector arm in demyelination, then what is the mechanism for activation of macrophages and why is not every inflammatory response in the CNS associated with demyelination? Activation may require 
lymphokines such as gamma interferon produced by activated $T$ cells, and it would occur as part of a local DTH response. Still to be identified are the antigen(s) recognized by the DTH-initiating $\mathrm{T}$ cells and the site where antigeh presentation takes place.

Either neural or viral antigens are reasonable possibilities. It was clearly demonstrated in studies of EAE that sensitization with MBP can produce an autoimmune disease often characterized by inflammation and demyelination. Recent studies showing that a relapsing form of EAE can be induced in mice by the transfer of $T$ cells sensitized to MBP bring this experimental model closer to MS. ${ }^{2}$ Furthermore, even the pathologic condition of these mice closely resembles that of MS. That MBP can be the relevant antigen in relapsing EAE is confirmed by the induction of the disease following transfer of $\mathrm{T}$-cell clones specific for the amino terminal portion of the molecule.?

Evidence of an enhanced cellular immune response to MBP in patients with MS is meager. One recent study of a large number of patients in which lymphoproliferation was used to measure reactivity to MBP indicated that the response was only slightly greater than that of a control group. ${ }^{10}$ The response to two other immunogenic components of myelin, myelin-associated glycoprotein and proteolipid protein, was not significantly different from that of the controls. In a separate study using T-cell clones generated from the CSF and blood of MS patients, none of the clones showed reactivity to MBP, whereas MBP-reactive clones were obtained from the CSF of patients with postinfectious encephalomyelitis." In the same study T-cell clones were also generated from the brain of one MS patient who had died, and again, none of the clones reacted to MBP. These findings suggest that even at the site of demyelination, MBP-reactive $T$ cells cannot be demonstrated. However, there are always questions regarding the age of the lesions sampled and the extent to which the clones reflect the repertoire of $T$ cells in the lesions. Even the small response of lymphocytes from the blood to MBP noted in some studies may not be specific for MS because similar responses have been observed in patients with other neurologic disorders.

A relationship between a virus and MS is supported by epidemiologic studies of MS and the elevated CSF antibody levels to many viruses. Additional support for this relationship comes from studies of Theiler's murine encephalonyelitis virus (TMEV) infection in mice. ${ }^{12}$ Neurologic signs related to demyelination occur one to several months following infection. The disease seems to be immunologically mediated, and susceptibility is influenced by the genetic makeup of the host. Genetic factors include genes in the MHC and at least one other set of genes outside the MHC and possibly coding for the beta-chain of the T-cell receptor. ${ }^{13}$ Susceptibility to disease correlates closely with the ability to generate a DTH response to TMEV. On the basis of these observations it was proposed that $\mathrm{T}$-cell recognition of virus still present within the CNS produces a DTH response that results in macrophage activation and subsequent demyelination. A process of this nature would not require persistence of infectious virus. Persistence of the viral genome and production of a single, even incomplete polypeptide that contains an epitope recognized by $T$ cells and not antibody would be sufficient to elicit a T-cell-mediated DTH response.

Many studies have examined the reactivity of lymphocytes from MS patients to various viral antigens. Most of these investigations used lymphoproliferation to measure reactivity, and the results were inconsistent. Recently, the generation of cytotoxic T cells (CTL) was examined in patients with MS and in appropriate control groups. ${ }^{14}$ A substantial number of patients had a significant reduction in their ability to generate measles-virus-specific CTL. In contrast, their ability to generate influenza-virus-specific CTL was the same as that of the control groups. Previous studies of measles-virusspecific CTL showed that they are CD4+ and that cytolysis is restricted by HLA 
class II antigens. These findings are distinct from those of CTL specific for many other viruses which are predominantly CD8+ and HLA class I restricted. The reduction in measles-virus-specific CTL is due to an approximately 5-to 10 -fold reduction in their precursor frequency in peripheral blood and not their total absence. ${ }^{15}$ The explanation for this finding remains uncertain. It could reflect a general reduction in a subset of CD4 + , although preliminary evidence indicates that other CD4 + HLA class II restricted CTL are not reduced. Therefore, the alteration probably reflects a difference in the initial infection or in the mechanisms responsible for maintaining long-term immunity. It is also possible that if this population of cells was directly involved in disease production, the reduced frequency in the peripheral blood could be due to migration and sequestration of these cells within the CNS. Presently, however, there does not appear to be a correlation between the reduced measles-virus-specific CTL and the clinical course. Thus, the possibility of T-cell recognition of viral antigens in the CNS, triggering an immune response with subsequent demyelination, has remained an attractive hypothesis, but proof for it is lacking.

A final possibility is that a viral infection produces sensitization to a neural antigen either by increasing the immunogenicity of that antigen or through cross-reactivity or mimicry. A subacute demyelinating disease was produced with a coronavirus, the JHM virus, and this EAE-like disease can be transferred with lymphocytes boosted in vitro with MBP in a manner similar to that used in the adoptive transfer studies of EAE. ${ }^{16}$ These findings indicate that infection with the JHM virus produces T-cell sensitization to MBP sufficient for subsequent transfer of an EAE-like disease. Sensitization to MBP following viral infections in humans was also reported. Studies of children with complicated or uncomplicated measles virus infections demonstrated that many children with measles encephalitis acquire a lymphoproliferative response to MBP. ${ }^{17}$ It is unknown if this sensitization is produced by the release of MBP or is due to a possible cross-reactivity. Some minor degrees of sequence homology were reported between several human viruses and MBP. ${ }^{18}$ The significance of these findings to MS is uncertain, but they do demonstrate that viral infections apparently can produce sensitization to neural antigens such as MBP.

\section{ANTIGEN PRESENTATION}

To produce a DTH type of response within the CNS the relevant antigen(s) needs to be presented to $T$ cells in the local environment. Cells that can function as antigenpresenting cells (APC) must be able to express MHC class II antigens on their membranes and to process antigens for presentation. Investigations over the last few years showed that two cell populations, endothelial cells and astrocytes, have the capability to express MHC class II antigens and can present MBP to sensitized T cells. ${ }^{19,20}$ Although neither cell population normally expresses significant quantities of Ia antigens, both can be induced to do so when factors such as gamma interferon are used. When endothelial cells are obtained from animals with EAE, they express MHC class II antigens, indicating that this expression has been induced in vivo. ${ }^{19}$ Studies of rats or mice susceptible or nonsusceptible to EAE showed that the ability to induce Ia antigens in cultured astrocytes from these animals correlates with susceptibility. ${ }^{21}$ Similar differences in the ability to induce HLA class II antigens in humans could represent one of the genetic factors operating in MS.

MHC class II antigens can also be induced in rat astrocytes by viral infection. Even inactivated virus seems to be capable of inducing these MHC antigens on 
astrocytes, especially on those derived from strains susceptible to EAE..$^{22}$ Therefore, a persistent infection could contribute to expression of class II antigens and, in turn, could lead to presentation of either viral or neural antigens. Importantly, this possibility suggests that a persistent infection could be instrumental to disease production triggered by $T$-cell reactivity to nonviral antigens. These events are more likely to occur in association with a diminished cellular immune response to the virus because the ability to eliminate the virus would be reduced.

\section{REGULATION}

One characteristic of MS is that it frequently follows a relapsing-remitting course, leading to the speculation that the disease may be related to an abnormality of suppressor cell function. In fact, deficiencies of suppressor cells were demonstrated on several experimental systems. ${ }^{23.24}$ The finding that the number of CD8 $+\mathrm{T}$ cells, which includes suppressor cells, is reduced during periods of worsening has been inconsistent. ${ }^{2 \varsigma, 26}$ However, functional studies showed that suppression is reduced during progression or exacerbation. These studies measured suppression as generated by mitogens such as concanavalin A or OKT3 which binds to the T-cell receptor. ${ }^{23}$ Similarly, the ability of CD8 $+\mathrm{T}$ cells to suppress Ig production following PWM stimulation is reduced, whereas other functional parameters of the CD8 $+\mathrm{T}$ cells such as CTL activity are normal. ${ }^{24} \mathrm{~A}$ subset of CD4 $+\mathrm{T}$ cells, the $2 \mathrm{H} 4$ cells, which act to induce suppression through the CD8 $+\mathrm{T}$ cell, is reduced in MS patients with active disease. ${ }^{27}$

The autologous mixed lymphocyte reaction (AMLR) is considered an important technique in studying immunoregulatory processes, and several investigators examined the AMLR in patients with MS. ${ }^{28-30}$ Generally, the response was increased during worsening. It has now been demonstrated that the greater response is due to a reduction in the generation of suppression which normally occurs in the AMLR. ${ }^{29}$

The significance of the various abnormalities of suppressor cell function is uncertain. Multiple sclerosis is not associated with multiple immunologic abnormalities, which casts some doubt on generalized suppressor cell deficiency, but episodic fluctuations in regulatory mechanisms could be involved in exacerbation. Alternatively, these changes in immunoregulation could result from the same process that triggers the immune response within the CNS. For example, up-regulation in HLA class II antigen expression could lead to increased reactivity in all of these in vitro tests and account for the apparent reduction in suppression. The significance of these findings is important to an understanding of the immune process in MS, and future studies hopefully will establish if these changes represent reduced suppression or enhanced reactivity secondary to the disease process.

\section{CONCLUSION}

Evidence linking the pathogenesis of MS to an immunologic process remains indirect and tentative. It is increasingly certain that the actual destruction of myelin is caused by activated macrophages. What is uncertain is the mechanism for activation 
of these macrophages. Although an immunologic mechanism seems most likely, it is still possible that activation could be a direct result of a viral infection. Extrapolation from observations made in various experiment models indicates that the most likely immunologic process is the induction of a DTH response with subsequent macrophage activation. Either a neural or viral antigen could trigger this response, and the genetic influence on susceptibility may reflect the genes coding for the HLA class II and Tcell receptor makeup necessary for recognition of the relevant antigen. Failure to demonstrate unique or enhanced $T$-cell reactivity is disturbing, but it could be due to the techniques used or to sequestration of this population within the CNS. Sequestration seems the least likely and has little support for experimental models.

It is now clear that both astrocytes and endothelial cells can function as antigenpresenting cells. The nonspecific induction of HLA class II antigens on either population, but especially endothelial cells by lymphokines such as gamma interferon, provides an attractive mechanism for presenting antigen to circulating cells and triggering a local immune response that leads to demyelination. Periodic increases in HLA class II expression caused by nonspecific processes such as viral infections could provide a reasonable explanation for the fluctuating course of the disease. With continued disease progression the local production of lymphokines may become sufficient to maintain an ongoing immune response.

Demyelination may be enhanced by local production of antibody capable of binding myelin. B cells with appropriate specificities migrating into the inflammatory site would find both antigen and lymphokines necessary for B-cell differentiation. The continued differentiation of $\mathbf{B}$ cells with production of antimyelin antibody could contribute to the conversion of the disease from a relapsing-remitting course to one of continued progression.

The major unanswered questions concern the antigen and the role of the genetic influence on the response. The ability of $T$ cells to recognize epitopes that consist of only several amino acids and that are not recognized by antibody may contribute to the difficulty in answering this question. Hopefully, techniques that allow manipulation of HLA genes and identification of characteristics of the T-cell receptor makeup, along with more specific immunologic methods, will contribute a greater understanding to these questions.

\section{REFERENCES}

1. Reder, A. T. \& B. G. W. ARnason. 1985. Immunology of multiple sclerosis. In Handbook of Clinical Neurology, Vol. 47. P. J. Vinken, G. W. Bruyn, \& H. C. Klawans, Eds.: 337-396. Elsevier, Amsterdam.

2. Raine, C. S., F. MohkTaRian \& D. E. MCFARI.IN. 1984. Adoptively transferred chronic relapsing experimental autoimmune encephalomyelitis in the mouse. Lab. Invest. 51: 534-546.

3. Tourtellotte, W. W., M. J. Wai.Sh, R. W. Baumhefner et al. 1984. The current status of multiple sclerosis intra-blood-brain-barrier $/ \mathrm{Ig} / \mathrm{g}$ synthesis. Ann. N.Y. Acad. Sci. 436: 52-67.

4. Ebers, C. G. 1984. Oligoclonal banding in MS. Ann. N.Y. Acad. Sci. 436: 206-212.

5. LISAK, R. P., B. ZWEIMAN, J. B. BURNS et al. 1984. Immune response to myelin antigen in multiple sclerosis. Ann. N.Y. Acad. Sci. 436: 221-230.

6. Prineas, J. W., E. E. Kwon, E. Cho \& L. R. Sharer. 1984. Continual breakdown and regeneration of myelin in progressive multiple sclerosis plaques. Ann. N.Y. Acad. Sci. 436: $11-32$. 
7. Raine, C. S., U. Traugott, F. Mohktakian et al. 1981. Augmentation of immunemediated demyelination by lipid haptens. Lab. Invest. 45: 174-182.

8. Adams, D. O. \& T. A. Hamilion. 1984. The cell biology of macrophage activation. Ann. Rev. Immunol. 2: 283-318.

9. Zamvil, S. S., P. A. Nelson, D. J. Mitche1.L. et al. 1985. Encephalitogenic T cell clones specific for myelin basic protein. J. Exp. Med. 162: 2107-2124.

10. Johnson, D., D. A. Hafler, R. J. Fallis et al. 1986. Cell-mediated immunity to myelin associated glycoprotein, proteolipid protein, and myelin basic protein in multiple sclerosis. J. Neuroimmunol. 13: 99-108.

11. Hafier, D. A., D. S. Benjamin, J. Burks, H. L. Weiner et al. 1987. Myelin basic protein and proteolipid reactivity of brain and cerebrospinal fluid-derived $T$ cell clones in multiple sclerosis and postinfectious encephalomyelitis. J. Immunol. 139: 68-72.

12. ClatCh, R. J., H. L. Lipton \& S. D. Miller. 1986. Characterization of Theiler's murine encephalomyelitis virus (TMEV)-specific delayed type hypersensitivity. J. Immunol. 136: 920-927.

13. Meivold, R. W., D. M. Jokinen, R. KNobler \& H. L. Lipton. 1987. Variations in genetic control of susceptibility to Theiler's murine encephalomyelitis virus(TMEV)induced demyelinating disease. J. Immunol. 138: 1429-1436.

14. Jacobson, S. J., M. L. Flerlage \& H. F. MCFARLAND. 1985. Impaired measles virusspecific cytotoxic $T$ cell responses in multiple sclerosis. J. Exp. Med. 162: 839-850.

15. McFarland, H. F., A. Goodman \& S. J. JaCOBSON. 1987. Virus specific cytotoxic T cells. Ann. N.Y. Acad. Sci. in press.

16. Watanabi, R., H. Wage \& V. TER Meulen. 1983. Adoptive transfer of EAE-like lesions from rats with coronavirus-induced demyelinating encephalomyelitis. Nature 305: $150-152$.

17. Johnson, R. T., D. E. Griffin, R. L. Hirsch et al. 1984. Measles encephalomyelitis-clinical and immunological studies. N. Engl. J. Med. 310: 137-141.

18. JAHNke, U., E. H. Fisher \& E. C. Al.vord. 1985. Sequence homology between certain viral proteins and proteins related to encephalomyelitis and neuritis. Science 229: 282 -284.

19. McCarron, R. M., M. SPATZ, O. KrMPSKi et al. 1986. Interaction between myelin basic protein-sensitized T lymphocytes and murine cerebral vascular endothelial cells. J. Immunol. 137: $3428-3435$.

20. Fontana, A., W. Fienz \& H. Wekerle. 1984. Astrocytes present myelin basic protein to encephalitogenic T cell lines. Nature 307: 273-276.

21. Massa, P. T., V. Ter Meulen \& A. Fontana. 1987. Hyperinductibility of Ia antigen on astrocytes correlates with strain specific susceptibility to experimental autoimmune encephalomyelitis. Proc. Natl. Acad. Sci. USA 84: 4219-4223.

22. Massa, P. T., R. Dorries \& V. TER Meulen. 1986. Viral particles induce la antigen expression on astrocytes. Nature 320: 543-544.

23. Antel, J. P., M. B. Bania, A. Reyda \& N. Cashman. 1986. Activated suppressor cell dysfunction in progressive multiple sclerosis. J. Immunol. 137: 137-141.

24. ANTel, J. P., M. K. Nickolas, M. B. Bania et al. 1986. Comparison of T8+ cellmediated suppressor and cytotoxic functions in multiple sclerosis. J. Neuroimmunol. 12: 215-224.

25. Weiner, H. L., D. A. Hafler \& R. J. Fall.js. 1984. T cell subsets in patients with multiple sclerosis. Ann. N.Y. Acad. Sci. 436: 281-290.

26. RICE, G. P., J. C. SIPE, S. L. BRAHERY el al. 1984. The failure of monoclonal antibodydefined lymphocyte subsets to monitor disease activity in multiple sclerosis. Ann. N.Y. Acad. Sci. 436: 271-280.

27. Morimoto, C., D. A. Hafler, H. L. Weiner et al. 1987. Selective loss of the suppressor inducer $T$ cell subset in progressive multiple sclerosis. N. Engl. J. Med. 316: 67-72.

28. Birnbaum, G. \& L. Kotjlinek. 1981. Autologous lymphocyte proliferation in multiple sclerosis and the effect of intravenous ACTH. Ann. Neurol. 9: 439-446.

29. Crisp, D. T., J. I. GREensteIN \& J. E. Kleiner. 1985. Regulation of the autologous mixed lymphocyte response in multiple sclerosis. Ann. Neurol. 18: 129.

30. Hafler, D. A., M. Buchsbaum \& H. L. Weiner. 1985. Decreased autologous mixed lymphocyte reaction in multiple sclerosis. J. Neuroimmunol. 9: 339-347. 